\title{
Making If-Then Plans Counteracts Learned Non-Use in Stroke Patients: A Proof-of- Principle Study
}

\author{
Michael K. MARQUARDT ${ }^{\mathrm{a}}$, Anna-Lisa COHEN ${ }^{\mathrm{b}}$, Peter M. GOLLWITZER ${ }^{\mathrm{a}, \mathrm{c}}$, Sam J. GILBERT ${ }^{\mathrm{d}}$ \\ and Christian DETTMERS ${ }^{\mathrm{e}}$ \\ ${ }^{a}$ Department of Psychology, University of Konstanz , Konstanz, Germany \\ ${ }^{b}$ Department of Psychology, Yeshiva University, New York, USA \\ ${ }^{c}$ Department of Psychology, New York University, New York, USA \\ ${ }^{d}$ Institute of Cognitive Neuroscience, University College of London, London, UK \\ ${ }^{e}$ Kliniken Schmieder Konstanz, Konstanz, Germany
}

Correspondence concerning this article should be addressed to Anna-Lisa Cohen, Department of Psychology, Yeshiva University, Belfer Hall, 2495 Amsterdam Avenue, New York 10033 US; Telephone: +1 212 960-5910; FAX: +1 212 960-5389; E-mail: acohen11@yu.edu

\begin{abstract}
Background: After stroke, the learned non-use of a paretic arm is a major obstacle to the improvement of hand function. Objective: We examined whether patients with a central paresis could profit from applying the self-regulation strategy of making if-then plans that specify situational triggers to using the paretic arm. Method: Seventeen stroke patients with a mild to moderate hand paresis were asked to perform a Simon task which is commonly used to study the enhanced executive control needed when there is a mismatch between stimulus (e.g., color) and response (e.g., location) features. We examined whether patients with hemiparesis would be able to reduce the Simon effect (i.e., responding slower to mismatch as compared to matched stimulus and response features) by creating new stimulus-response associations via if-then plans. Results: A significant Simon effect was observed in both the affected and the non-affected arm for control trials. However, there was no longer a significant Simon effect for the critical trials prepared by forming if-then plans. This led to a significant stimulus x compatibility interaction effect for the affected arm and a marginally significant interaction effect for the non-affected arm. Making if-then plans was effective for eliminating or at least reducing the Simon effect for the affected and the non-affected arm, respectively. Conclusion: This observation opens a potential new route to improving stroke rehabilitation. If-then plans may qualify as a viable strategy to overcome the learned non-use of the affected arm. Further research is now required to develop and test therapeutic measures based on this proof-of-principle.
\end{abstract}

Key-words: implementation intentions, rehabilitation, hemiparesis, stroke, learned nonuse 


\section{Introduction}

After a stroke, approximately $80 \%$ of patients experience an acute paresis and in about $40 \%$ a chronic hemiparesis remains (i.e., partial paralysis of the body and extremities due to brain lesion; Writing et. al, 2016). Hemiparesis is associated with high costs as it requires intensive treatment in the hospital, long-term care, loss of productivity and income. Per annum costs to treat U.S. patients with hemiparesis have been estimated to be almost twice as high as for those stroke patients without hemiparesis (Zorowitz, Chen, Tong, Laouri, 2009). Long-term benefits from rehabilitation and particularly transfer of improved capacities into daily life are required. Consequently, it is important to develop time and cost effective treatments to improve motor control in hemiparesis patients. The present study investigates whether teaching patients with hemiparesis to plan out motor control in advance could potentially yield an improvement in their symptoms.

Stroke patients with arm hemiparesis (i.e., impaired control of an arm) tend to use the less affected arm for various everyday activities as this allows the individual to avoid the increased effort required to use the affected arm. As a consequence, a strong habit develops of predominantly using the nonaffected rather than the affected arm. This phenomenon has been referred to as "learned non-use" (Taub, Uswatte, Mark, \& Morris, 2006). Besides physiotherapy (i.e., practicing the use of the affected arm), constraint-induced-movement therapy (CIMT) is an evidence-based therapy to deal with "learned non-use" (Taub et al., 1993; 2013; Wolf et al., 2013). In CIMT, the less affected hand is restricted by a sling or a similar device, forcing the patient to use the impaired hand. CIMT is highly effective and has had an enormous impact on physiotherapy and neurorehabilitation in general. Widespread use of its original version in rehabilitation settings, however, is limited due to the laborious nature of the therapy (Corbetta, Sirtori, \& Gatti, 2010; Dada \& Sanya, 2012). The original version of CIMT training is indicated for at least six hours a day on ten consecutive days (Taub et al., 1993). More recent studies suggest that application of less training time and a shorter restriction period of the less-affected arm (two to four hours /weekday; "modified CIMT") is still effective. A recent review could not demonstrate different effect sizes between modified CIMT and the original form (Kwakkel et al., 2015). 
In the current study, we considered whether there might be a more convenient way to avoid the negative consequences associated with learned non-use of the affected arm. In the psychology of action literature, it is suggested that making explicit plans in the service of higher order goals can facilitate action control. In particular it is argued that furnishing goals with implementation intentions (i.e., if-then plans) transforms effortful top-down control by goals into automatic bottom-up control by prespecified critical situations (Gollwitzer \& Bargh, 1996; Morsella, Bargh, \& Gollwitzer, 2009; Gollwitzer, 1993, 1999). In other words, implementation intentions link anticipated critical future situations with goal-directed responses, which commits the individual to respond in a predetermined way. Implementation intentions take the format of "If Situation X is encountered, then I will perform Response Y!" It is important to distinguish implementation intentions from the more simple structure of goal intentions: “I intend to attain Z!" Implementation intentions are formed in the service of goal intentions as they specify when, where, and how a goal-directed response is to be executed. A metaanalysis by Gollwitzer and Sheeran (2006) found that forming implementation intentions has a medium-to-large effect on the rate of goal attainment. A heightened cognitive accessibility of the specified critical situation as well as a strengthened associative link between the specified situation and the appropriate response qualify as the underlying mechanisms, allowing for automatic action control when the critical situation is encountered.

Indeed, previous research shows that implementation intentions were effective in controlling habitual responses (Adriansee et al., 2011; Gollwitzer, 2014; Gollwitzer et al., 2005; Stewart \& Payne, 2008; Schweiger-Gallo et al., 2009). Making if-then plans thus creates an "instant habit" for initiating the antagonistic wanted response that can override the initiation of the unwanted habitual response (Adriansee et al., 2011). Therefore, the present research will explore whether stroke patients with arm hemiparesis can use if-then planning to facilitate the use of the affected over the unaffected arm.

In the present study, we used a task which is well suited for studying the control of habitual actions, the Spatial Simon task (Simon \& Rudell, 1967). In this classification task, the relevant stimulus dimension is a non-spatial dimension such as color. For example, the participant is asked to make a left key press if the stimulus is red and a right key press if it is green (Simon, 1990). The location of the stimulus (left or right side of the computer screen) is the irrelevant stimulus dimension. Much re- 
search has shown that responses are faster when the stimulus location corresponds to the location of the assigned response (e.g., red stimulus on the left) as compared to when stimulus location and location of response are non-corresponding (e.g., red stimulus on the right). This phenomenon is commonly referred to as the Simon effect (see Lu \& Proctor, 1995). Even though participants are instructed to respond on the basis of the stimulus dimension (i.e., color) and ignore the irrelevant dimension (i.e., location on left or right of computer screen), participants find it difficult to do so; the irrelevant dimension of location interferes with performance.

The Simon effect is a prime example of automatic response activation based on long-term associations (i.e., automatic triggering of a habitual action). However, previous research has shown that the Simon effect can be reduced by forming implementation intentions (e.g., Cohen, Bayer, Jaudas, \& Gollwitzer, 2008; Miles \& Proctor, 2008). For example, in Experiment 2 of Cohen et al. (2008), participants performed a Simon task that was based on an experimental design used by Hommel (1993, Experiment 1). Participants had to decide whether the pitch of a tone (that occurred either on the left or right ear) was high or low by pressing a left or right key. Thus, in order to control the habitual response of pressing the left button when the tone is presented on the left side, participants in the implementation intention condition formed an if-then plan: "And if I hear the low tone on the left side, then I will press the right key especially fast!” In contrast, participants in the goal intention condition formed the mere goal: "I'll respond to the tones by pressing the respective button as fast as possible!" As action initiation via implementation intentions is assumed to be automatic, a reduced Simon effect was predicted and observed for participants in the implementation intention but not the goal intention condition. This interpretation is in line with a more recent finding that people can indeed counteract the Simon effect by strong stimulus-response associations formed on the basis of instructions (Theeuwes, Liefooghe, \& De Houwer, 2014).

In the present study, we investigated whether patients with hemiparesis are able to use implementation intentions to facilitate motor responses, using a Simon task. Furthermore, we explored if this self-regulatory technique is effective for facilitating motor responses in the paretic arm. Such a result would imply that implementation intentions may be useful as a method to promote the use of residual movement capacity in the paretic arm, perhaps improving functional outcome after stroke (proof-of- 
principle). The Simon task is particularly appropriate for this purpose because it involves conflict between a habitual response (respond according to the location of the stimulus) and a non-habitual response (respond according to a different stimulus dimension). In this way, it may be considered analogous to the conflict in patients with hemiparesis between habitual responding with their non-affected arm versus non-habitual responding with their affected arm. If implementation intentions are effective for overcoming the conflict presented by the Simon task, this would suggest that they could also facilitate patients' non-habitual use of their affected arm.

\section{Methods}

\subsection{Sample}

Over the course of one year qualifying patients (i.e., with mild-to-moderate arm hemiparesis) who arrived at a neurological rehabilitation clinic in Germany were informed about the study by their treating physicians and offered to participate. Participants who were not able to follow instructions due to impairments were excluded, such as neglect, visual inattention, language processing deficits, cognitive impairments. Of the 19 patients recruited, two were not able to follow instructions. The sample included 17 stroke patients ( 9 women; age: $M=56.76, S D=11.72$ ) with a central arm paresis ( 5 leftsided, 12 right-sided hand paresis) but sufficient hand control to press computer keys with the index fingers. The mean time since the stroke was 23.58 months $(S D=46.24)$ : 10 patients 4-6 weeks (subacute), and 7 patients 2-12 years. As indicated by the National Institute of Health Stroke Scale (NHISS; Goldstein, Bartels, \& Davis, 1989. all patients had minor impairments due to hemiparesis (NHISS Score $<4$ ), except for one patient who showed a moderate impairment (NHISS Score $=5$ ). Based on the Nine-Hole-Peg (NHP) test (Jebsen, Taylor, Trieschmann, Trotter, \& Howard, 1969; Mathiowetz, Weber, Kashman, et al., 1984), 11 patients showed mild hand paresis (NHP time $<11$ s), 5 patients moderate (NHP time $<50$ s), and one patient severe hand paresis $(\mathrm{NHP}=99 \mathrm{~s})$. Six patients had a light, incomplete hypoesthesia. One patient had a hemorrhagic infarction, and 16 were ischemic. One patient had an infarct in the pons, the others had partial infarction of the middle cerebral artery (MCA). For all patients, the respective attending physicians reported a critical learned-nonuse of the affected arm. The study protocol was in accordance with the Declaration of Helsinki and approved by the ethical board of the University of Konstanz. All patients gave informed consent. 


\subsection{Apparatus and Stimuli}

The Simon task was programmed using Presentation ${ }^{\circledR}$ software (www.neurobs.com) and presented on a Laptop-computer with a 14-inch LCD Monitor; viewing distance was $60 \mathrm{~cm}$. The target stimuli were arrows of four different colors (blue, green, red, and yellow) pointing either to the right or to the left (see Figure 1). In a given trial, an arrow of one of the four colors was presented, pointing to one direction. Participants responded by pressing either a key located to the left (left alt) or right (control) of the bottom row of the keyboard with their left or right index finger, respectively. For each trial a central black fixation cross preceded each arrow.

\subsection{Procedure}

The Simon task involved pressing either a left (left alt key) or right (control key) in response to the color of the task stimuli. For example, participants were instructed that for blue or green arrows, they need to press the left computer key and, for yellow or red arrows, they need to press the right computer key. The arrows could be facing right or left which was irrelevant to task instructions. That is, the direction that arrows were pointing (left or right) was the irrelevant stimulus dimension. As described previously, the Simon effect is the phenomenon in which responses are faster and more accurate when the direction of a stimulus (arrow pointing left or right) corresponds to the location of an assigned response (left or right computer key press). Performance is slower and more error prone when the stimulus-response correspondence is noncorresponding (arrow pointing left but requires a right computer key press). The challenge for participants in our study was to ignore the irrelevant stimulus dimension (arrow pointing left or right) and solely attend to the color of stimuli. The mapping of the color to the correct response location was counterbalanced across participants.

Practice and test blocks combined consisted of 256 trials in total. A session had the following course of events: At the onset, participants were provided with the goal instruction on how to respond to stimuli, followed by a first practice block with 16 trials including each possible combination of color and arrow-direction presented twice. After this first practice block, participants received feedback regarding incorrect responses. The subsequent second practice block consisted of 80 trials presenting each possible combination of color and arrow direction ten times. This second block provided additional practice and served to reduce earlier fluctuation in response times. After this block of trials, 
participants received feedback on their performance indicating the percentage of correct and wrong responses, followed by a 2-minute break.

Each trial began with the fixation cross presented for $1000 \mathrm{~ms}$ followed by an arrow presented for $320 \mathrm{~ms}$ in one of the four colors pointing to the left or right. Participants made a left or right response depending on the color. Inter-trial intervals randomly ranged between $3 \mathrm{~s}$ and $7 \mathrm{~s}$ (i.e., 3, 4, 5, 6, 7). We used a within-participants experimental design. For the first block of test trails, participants always received goal intention instructions, and implementation intention instructions were always given for the second block of test trials. This order was not counterbalanced because we worried that participants who had learned to form implementation intentions and experienced their beneficial effects might spontaneously furnish goal intentions with implementation intentions (see Cohen et al., 2008; Miles \& Proctor, 2008).

Preceding the first test block of trials, participants received the goal instructions that read, for example: "For a blue arrow or a green arrow you should press the left key as fast as possible. For a yellow arrow or a red arrow you should press the right key as fast as possible." Participants read the goal instructions three times aloud and then worked on the first block of 80 test trials with each possible combination of color and arrow direction presented ten times. In corresponding trials, the arrows pointed to the same side as specified in the goal instructions (e.g., blue and green arrows to the left, yellow and red arrows to the right) and in non-corresponding trials to the opposite direction (e.g., blue and green arrows to the right, yellow and red arrows to the left; see Figure 1). Following this first set of test trials, participants received feedback and took a 2 min break. Before the next set of test trials began, participants repeated the goal instructions aloud before they read the implementation intention instructions three times. The implementation intention read, for instance: "Each time, when the green arrow points to the right, then I instantly will press the left key. Each time, when the red arrow points to the left, then I instantly will press the right key." Instructions were counterbalanced for each arrow color and arrow direction with the constraint that implementation intentions always addressed two non-corresponding color-arrow combinations with the two arrows pointing in opposite directions. This ensured that responses with non-affected and affected arms on corresponding and non-corresponding stimuli had to be performed equally often. 
We defined critical trials as those that showed an arrow with the color specified in the participants' implementation intentions; all other trials were considered to be control trials. For example, a critical trial would be those trials that included the stimuli stipulated in the implementation intention. If the implementation intention was "Each time, when the green arrow points to the right, then I instantly will press the left key. Each time, when the red arrow points to the left, then I instantly will press the right key", then we expected that participants would show higher performance on these noncorresponding trials compared to trials with stimuli not specified in the implementation intention. The experiment lasted between 45-60 min after which time patients were debriefed and thanked for their participation.

\section{Results}

\subsection{Response Times (RT)}

\subsubsection{First block of test trials}

Trials in which RTs were less than $200 \mathrm{~ms}$ or were more than 2 SDs from the mean response time were deleted ( $4.1 \%$ of all responses). For the first block of trials, a 2 (Arm: affected vs. non-affected) x 2 (Stimulus: critical vs. control) x 2 (Compatibility: corresponding vs. non-corresponding) withinfactors ANOVA was conducted on RTs. No interaction effects were found, all $F \mathbf{s}<1, n s$. However, the expected Compatibility main effect emerged, $F(1,16)=17.21, p=.001, \eta_{\mathrm{p}}{ }^{2}=.52$, indicating that participants responded faster in corresponding trials than in non-corresponding trials. No other main effects were observed, all $F \mathbf{s}<1, n s$ (see Table 1, Block 1).

\subsubsection{Second block of test trials}

To test whether implementation intentions reduced response times for the specified critical noncorresponding stimuli, a 2 (Arm: affected vs. non-affected) x 2 (Stimulus: critical vs. control) x 2 (Compatibility: corresponding vs. non-corresponding) ANOVA on RTs was conducted for the second test block of trials. No three-way interaction effect was found, $F(2,16)<1, n s$, but the expected twoway Stimulus (critical vs. control) x Compatibility (corresponding vs. non-corresponding) interaction emerged, $F(1,16)=6.52, p=.02, \eta_{\mathrm{p}}^{2}=.29$. No other two-way interaction, all $F \mathrm{~s}<1$, $n s$, or main effects, all $F_{\mathrm{s}}<1, n s$, were observed other than a Compatibility main effect, $F(1,16)=9.16, p<.01, \eta_{\mathrm{p}}{ }^{2}$ $=.36$. 
As expected, and replicating the Simon effect, planned one-tailed comparisons revealed that participants responded faster to control stimuli in corresponding $(M=624 \mathrm{~ms} ; S E=38)$ than in noncorresponding trials $(M=662 \mathrm{~ms} ; S E=41), t(16)=4.49, p<.001$. More importantly, participants responded faster to critical stimuli in non-corresponding trials $(M=635 \mathrm{~ms}, S E=32)$ than to control stimuli in non-corresponding trials, $t(16)=1.86, p=.04$. No differences were found for critical stimuli in non-corresponding and corresponding trials $(M=629 \mathrm{~ms} ; S E=36)$ as well as for critical and control stimuli in corresponding trials, all $t \mathrm{~s}<1, n s$ (Table 1, Block 1, Critical and Control).

To test whether the predicted implementation intention effects similarly appeared for the affected as well as non-affected arm, two separate 2 (Stimulus: critical vs. control) x 2 (Compatibility: corresponding vs. non-corresponding) ANOVAs, one for each arm, were conducted. The analysis for the affected arm revealed the expected Stimulus x Compatibility interaction effect, $F(1,16)=4.76, p=.04$, $\eta_{\mathrm{p}}{ }^{2}=.23$, and a Compatibility main effect, $F(1,16)=4.30, p=.03, \eta_{\mathrm{p}}{ }^{2}=.21$. Planned one-tailed comparisons replicated the previously found pattern: Participants responded faster to control stimuli in corresponding $(M=634 \mathrm{~ms} ; S E=54)$ than in non-corresponding trials $(M=673 \mathrm{~ms} ; S E=56), t(16)=$ $3.49, p<.01$. Participants also responded marginally faster to critical stimuli in non-corresponding trials $(M=644 \mathrm{~ms} ; S E=46)$ than to control stimuli in non-corresponding trials, $t(16)=1.60 ; p=.06$. No differences were found for critical stimuli in non-corresponding and corresponding trials $(M=$ $641 \mathrm{~ms} ; S E=53$ ) as well as for critical and control stimuli in corresponding trials, all $t \mathrm{~s}<1, n s$.

A parallel 2 (Stimulus: critical vs. control) x 2 (Compatibility: corresponding vs. noncorresponding) ANOVA analysis for the non-affected arm did not reach significance, $F(1,16)=3.63$, $p=.07, \eta_{\mathrm{p}}{ }^{2}=.19$, but revealed a Compatibility main effect $F(1,16)=7.01, p=.02, \eta_{\mathrm{p}}{ }^{2}=.31$. However, planned one-tailed comparisons also replicated the previously found pattern: Participants responded faster to control stimuli in corresponding $(M=614 \mathrm{~ms} ; S E=24)$ than in non-corresponding trials $(M$ $=649 \mathrm{~ms} ; S E=28), t(16)=3.64, p=.001$. Participants also responded marginally faster to critical stimuli in non-corresponding trials $(M=625 \mathrm{~ms} ; S E=24)$ than to control stimuli in non-corresponding trials, $t(16)=1.42 ; p=.09$. No differences were found for critical stimuli in non-corresponding and corresponding trials $(M=617 \mathrm{~ms} ; S E=23)$ as well as for critical and control stimuli in corresponding trials, all $t \mathrm{~s}<1, n s$. 
In sum, a significant Simon effect was observed in both arms for control trials. However, there was no longer a significant Simon effect for critical trials, which were associated with implementation intentions. Importantly, this result demonstrates that implementation intentions reduced the Simon effect (i.e., response times for non-corresponding trials as compared to corresponding trials) for the affected arm by $M=37 \mathrm{~ms}(S E=17)$ as well as for the non-affected arm by $M=28 \mathrm{~ms}(S E=15)$ (see the last line in Table 1). This led to a Stimulus x Compatibility interaction that was significant for the affected arm and marginally-significant for the non-affected arm. Apparently, implementation intentions were effective in reducing the Simon effect for both arms.

\subsection{Percentage of errors (PE)}

\subsubsection{First block of test trials}

The overall percentage of errors (PE) was low for the affected $\operatorname{arm}(M=2.77 \% ; S E=1.23)$ and the non-affected $\operatorname{arm}(M=4.60 \%, S E=1.63)$. A 2 (Arm: affected vs. non-affected) $x 2$ (Stimulus: critical vs. control) x 2 (Compatibility: corresponding vs. non-corresponding) between-factors ANOVA revealed an Arm (affected vs. non-affected) x Compatibility (corresponding vs. noncorresponding) interaction effect, $F(1,16)=5.53, p=.03, \eta_{\mathrm{p}}{ }^{2}=.26$, and main effects of Arm, $F(1,16)$ $=5.18, p=.04, \eta_{\mathrm{p}}{ }^{2}=.25$, and Compatibility, $F(1,16)=5.18, p=.04, \eta_{\mathrm{p}}{ }^{2}=.25$. Post-hoc comparisons revealed that participants made more errors with the non-affected arm in non-corresponding trials than in corresponding trials, $t(16)=2.78, p=.01$. No such difference was found for the affected arm, $t(16)$ $=1.38, n s$. Participants made more errors with the non-affected arm than with the affected arm in noncorresponding trials, $t(16)=2.46 ; p=.03$. No such difference was found for corresponding trials, $t<$ 1, ns (see Table 1, Block 1).

\subsubsection{Second block of test trials}

PE was low for the affected arm $(M=5.26 \% ; S E=1.61)$ and the non-affected arm $(M=4.03 \%$, $S E=1.48)$. A 2 (Arm: affected vs. non-affected) x 2 (Stimulus: critical vs. control) x 2 (Compatibility: corresponding vs. non-corresponding) ANOVA revealed a three-way interaction effect, $F(1,16)=$ $7.01, p=.03, \eta_{\mathrm{p}}^{2}=.30$. Pairwise comparisons indicated a significant difference $(\mathrm{p}=.049)$ between performance in the critical non-corresponding trials for the affected arm $(\mathrm{M}=3.53 \%)$ compared to the control non-corresponding trials for the affected arm $(\mathrm{M}=10.62 \%)$. No other pairwise comparisons 
turned out to be significant. There was also a main effect of Stimulus, $F(1,16)=4.73, p=.05, \eta_{\mathrm{p}}{ }^{2}=$ .23 , showing a lower percentage of errors for critical versus control trials. No other effects were found, all $F \mathrm{~s}(1,16)<1, n s$. To test whether decreased response times might have been due to response timeerror rate tradeoffs, two separate 2 (Stimulus: critical vs. control) x 2 (Compatibility: corresponding vs. non-corresponding) ANOVAs for each arm were conducted. Neither the analysis for the affected arm nor the analysis for the non-affected arm revealed any interaction or main effects, all $F \mathrm{~s}(1,16)<$ 3.71, all $p \mathrm{~s}>.07$ (see Table 1, Block 2, Critical and Control).

\section{Discussion}

We examined whether patients with mild to moderate arm hemiparesis could benefit from the use of implementation intentions (i.e., if-then plans) to facilitate non-habitual motor responses in a controlled experimental setting. We explored this research question by using a Simon task where problems of action control due to habitual responding are prevalent. Results showed that stimuli specified in if-then plans were associated with a reduced Simon effect as compared to unspecified (control) stimuli. Importantly, patients with arm hemiparesis were able to successfully form and execute their if-then plans, benefitting action control by their affected arm. This suggests that making if-then plans enables patients with hemiparesis to willfully reduce habitual responding. This is a first indication of the possibility that a similar technique might allow patients with hemiparesis to reduce habitual use of their non-affected arm rather than making use of their residual motor capacity in the affected arm.

After a stroke, insufficient use and training of the affected hand becomes a central concern. This is thought to reflect learned suppression of the affected arm, which may persist even after motor recovery has occurred (Sterr, Freivogel, \& Schmalohr, 2002). Consistent with this view, even though all participants in the present study showed minor or moderate impairment due to hemiparesis, motor function as assessed in the Simon task was no worse for the affected than the non-affected arm. Indeed, accuracy in Block 1 was higher for the affected than the non-affected arm. This hints at a speedaccuracy trade-off, given that RTs were also slightly higher for the affected arm. However, no strong conclusions can be drawn here because this RT difference was not statistically significant.

Today, one of the most influential treatments for hemiparesis is constraint-induced movement therapy (CIMT; Taub et al., 1993, 2006). While the original version with six hours of suggested motor 
training of the affected and restraint use of the non-affected arm (and still three hours with the modified CIMT) can be daunting and also difficult to implement, the efficacy of the major "ingredients" of CIMT like intensive repetitive motor training and shaping of the activities of everyday life (e.g., by making these activities gradually more difficult) is high. Transferring therapeutic gains from the treatment setting to the daily life was found to increase the gains by the factor of 2.4. (Taub et al., 2013). Still, teaching patients to form respective implementation intentions could provide a new, highly convenient way to promote controlled use of the affected arm while counteracting learned-nonuse. If supported by future research testing actual clinical relevance and application, this self-regulation strategy might complement conventional techniques to improve motor control in stroke patients.

Studies of use-dependent plasticity indicate that the sensorimotor system adapts as a result of skilled activities such as reading Braille or playing violin (Elbert et al., 1995; Pascual-Leone et al., 1995). Conversely, motor restriction can lead to rapid sensorimotor adaptation. For example, by using transcranial magnetic stimulation it could be shown that short-term motor restriction decreased motor cortical excitability(Facchini et al., 2002). Moreover, 48 hours of hand immobilization were found to lead to impaired motor imagery for the immobilized hand (Toussaint \& Meugnot, 2013). However, there was no effect on visual imagery, suggesting that motor restriction may have selective effects on motor representations. The brain systems involved in these motor representations are likely to be distinct from those involved in action control by implementation intentions. For example, fMRI evidence suggests that non-motor brain regions such as rostral prefrontal cortex (BA10) play an important role in action control by if-then plans (Gilbert et al., 2009). One of the potential advantages of an implementation intention strategy, therefore, is that it could potentially utilize spared visual and cognitive control mechanisms in order to boost motor function. This would especially be the case if the "if" component of the if-then plan was specified with respect to visual rather than motor triggers.

Despite some encouraging results, the current research must be perceived as a first step to investigate the potential of implementation intentions for treatment of patients suffering from hemiparesis. Several limitations must be addressed in further research to support the clinical relevance and applicability of implementation intentions for therapeutic use. First, the current study was implemented with a small sample with heterogeneity of stroke localization which was due to the primary selection criteri- 
on of being able to follow task instructions. The selection criterion of mild-to-moderate arm hemiparesis was based on clinical judgement rather than an a priori score obtained via a validated instrument such as the Motor Activity Log. However, one might note that heterogeneity as well as varying average time since onset suggests that the effect is not restricted to a particular "sensitive period" or type of stroke (Zeiler et al. 2016). Second, the current study tested short term reaction time effects and did not concentrate on therapeutic long-term benefits. Third, the study was implemented within a controlled experimental setting not reflecting the real-life environments where therapeutic treatment needs to be effective. To ensure that the found implementation intention effects are meaningful and generalizable for clinical application, further research is thus required to test whether our findings can be used to translate current insights into a meaningful therapeutic approach. In sum, the current research provides an early proof-of-concept that the use of if-then plans could turn out to be a simple and efficient means to improve therapy of patients suffering from hemiparesis after stroke and to counteract learned non-use. 


\section{References}

Adriaanse, M. A., Gollwitzer, P. M., De Ridder, D. T., De Wit, J. B., \& Kroese, F. M. (2011). Breaking habits with implementation intentions: A test of underlying processes. Personality and Social Psychology Bulletin, 37, 502-513. doi:10.1177/0146167211399102.

Cohen, A. L., Bayer, U. C., Jaudas, A., \& Gollwitzer, P. M. (2008). Self-regulatory strategy and executive control: Implementation intentions modulate task switching and Simon task performance. Psychological Research, 72, 12-26. doi:10.1007/s00426-006-0074-2

Corbetta, D., Sirtori, V., Moja, L., \& Gatti, R. (2010). Constraint-induced movement therapy in stroke patients: systematic review and meta-analysis. European Journal of Physical and Rehabilitation Medicine, 46, 537-544.

Dada, O. O., \& Sanya, A. O. (2012). Constraint-induced movement therapy: Determinants and correlates of duration of adherence to restraint use among stroke survivors with hemiparesis. Disability, CBR \& Inclusive Development, 22, 15-28. doi:10.5463/dcid.v22i3.84.

Elbert, T., Pantev, C., Wienbruch, C., Rockstroh, B., \& Taub, E. (1995). Increased cortical representation of the fingers of the left hand in string players. Science, 270, 305-307. doi:10.1126/science. 270.5234 .305

Facchini, S., Romani, M., Tinazzi, M., \& Aglioti, S. M. (2002). Time-related changes of excitability of the human motor system contingent upon immobilization of the ring and little fingers. Clinical Neurophysiology, 113, 367-375. doi:10.1016/S1388-2457(02)00009-3

Gilbert, S. J., Gollwitzer, P. M., Cohen, A. L., Oettingen, G., \& Burgess, P. W. (2009). Separable brain systems supporting cued versus self-initiated realization of delayed intentions. Journal of Experimental Psychology: Learning, Memory, and Cognition, 35, 905-915. doi:10.1037/a0015535

Goldstein, L. B., Bertels, C., \& Davis, J. N. (1989). Interrater reliability of the NIH stroke scale. Archives of Neurology, 46, 660-662. doi:10.1001/archneur.1989.00520420080026 
Gollwitzer, P. M. (1993). Goal achievement: The role of intentions. European Review of Social Psychology, 4, 141-185. doi:10.1080/14792779343000059

Gollwitzer, P. M. (1999). Implementation intentions: strong effects of simple plans. American Psychologist, 54, 493-503. doi:10.1037/0003-066X.54.7.493

Gollwitzer, P. M. (2014). Weakness of the will: Is a quick fix possible? Motivation and Emotion, 38, 305-322. doi:10.1007/s11031-014-9416-3.

Gollwitzer, P. M., \& Bargh, J. A. (Eds.). (1996). The psychology of action: Linking cognition and motivation to behavior. New York: Guilford Press.

Gollwitzer, P. M., Bayer, U., \& McCulloch, K. (2005). The control of the unwanted. In R. Hassin, J. Uleman, \& J. A. Bargh (Eds.), The new unconscious (pp. 485-515). Oxford: Oxford University Press.

Gollwitzer, P. M., \& Sheeran, P. (2006). Implementation intentions and goal achievement: A metaanalysis of effects and processes. Advances in Experimental Social Psychology, 38, 69-119. doi:10.1016/S0065-2601(06)38002-1

Hommel, B. (1993). Inverting the Simon effect by intention. Psychological Research, 55, 270-279.

Jebsen, R. H., Taylor, N., Trieschmann, R. B., Trotter, M. J., \& Howard, L. A. (1969). An objective and standardized test of hand function. Archives of Physical Medicine and Rehabilitation, 50, 311-319.

Kwakkel, G., Veerbeek, J. M., van Wegen, E. E., \& Wolf, S. L. (2015). Constraint-induced movement therapy after stroke. The Lancet Neurology, 14, 224-234. doi: 10.1016/S1474-4422(14)701607

Lu, C. H., \& Proctor, R. W. (1995). The influence of irrelevant location information on performance: A review of the Simon and spatial Stroop effects. Psychonomic Bulletin \& Review, 2, 174207. doi:10.3758/BF03210959 
Mathiowetz, V., Weber, K., Volland, G., \& Kashman, N. (1984). Reliability and validity of grip and pinch strength evaluations. The Journal of Hand Surgery, 9, 222-226. doi:10.1016/S03635023(84)80146-X

Miles, J. D., \& Proctor, R. W. (2008). Improving performance through implementation intentions: Are preexisting response biases replaced? Psychonomic Bulletin \& Review, 15, 1105-1110. doi: 10.3758/PBR.15.6.1105

Morsella, E., Bargh, J. A., \& Gollwitzer, P. M. (Eds.). (2009). Oxford handbook of human action. New York: Oxford University Press.

Pascual-Leone, A., Wassermann, E. M., Sadato, N., \& Hallett, M. (1995). The role of reading activity on the modulation of motor cortical outputs to the reading hand in Braille readers. Annals of Neurology, 38, 910-915. doi:10.1002/ana.410380611

Schweiger-Gallo, I., Keil, A., McCulloch, K. C., Rockstroh, B., \& Gollwitzer, P. M. (2009). Strategic automation of emotion regulation. Journal of Personality and Social Psychology, 96, 11-31. doi:10.1037/a0013460

Simon, J. R. (1990). The effects of an irrelevant directional cue on human information processing. Advances in Psychology, 65, 31-86. doi:10.1016/S0166-4115(08)61218-2

Simon, J. R., \& Rudell, A. P. (1967). Auditory SR compatibility: The effect of an irrelevant cue on information processing. Journal of Applied Psychology, 51, 300-304. doi:10.1037/h0020586

Sterr, A., Freivogel, S., \& Schmalohr, D. (2002). Neurobehavioral aspects of recovery: Assessment of the learned nonuse phenomenon in hemiparetic adolescents. Archives of Physical Medicine and Rehabilitation, 83, 1726-1731. doi:10.1053/apmr.2002.35660

Stewart, B. D., \& Payne, B. K. (2008). Bringing automatic stereotyping under control: Implementation intentions as efficient means of thought control. Personality and Social Psychology Bulletin, 34, 1332-1345. doi:10.1177/0146167208321269. 
Taub, E., Miller, N. E., Novack, T. A., Cook 3rd, E. W., Fleming, W. C., Nepomuceno, C. S., ... \& Crago, J. E. (1993). Technique to improve chronic motor deficit after stroke. Archives of Physical Medicine and Rehabilitation, 74, 347-354.

Taub, E., Uswatte, G., Mark, V. W., \& Morris, D. M. (2006). The learned nonuse phenomenon: implications for rehabilitation. Europa Medicophysica, 42, 241-55.

Taub, E., Uswatte, G., Mark, V. W., Morris, D. M., Barman, J., Bowman, M. H., ... \& Bishop-McKay, S. (2013). Method for enhancing real-world use of a more affected arm in chronic stroke transfer package of constraint-induced movement therapy. Stroke, 44, 1383-1388. doi:10.1161/STROKEAHA.111.000559

Theeuwes, M., Liefooghe, B., \& De Houwer, J. (2014). Eliminating the Simon effect by instruction. Journal of Experimental Psychology: Learning, Memory, and Cognition, 40, 1470-1480.. doi:10.1037/a0036913

Toussaint, L., \& Meugnot, A. (2013). Short-term limb immobilization affects cognitive motor processes. Journal of Experimental Psychology: Learning, Memory, and Cognition, 39, 623-632. doi: $10.1037 / \mathrm{a} 0028942$

Wolf, S. L., Winstein, C. J., Miller, J. P., Taub, E., Uswatte, G., Morris, D., ... \& Excite Investigators. (2006). Effect of constraint-induced movement therapy on upper extremity function 3 to 9 months after stroke: the EXCITE randomized clinical trial. JAMA, 296, 2095-2104. doi:10.1001/jama.296.17.2095

Writing, G. M., Mozaffarian, D., Benjamin, E. J., Go, A. S., Arnett, D. K., Blaha, M. J., ... \& Fullerton, H. J. (2016). Heart Disease and Stroke Statistics-2016 Update: A report from the American Heart Association. Circulation, 133, e38-e360. doi: 10.1161/CIR.0000000000000350.

Zeiler, S. R., Hubbard, R., Gibson, E. M., Zheng, T., Ng, K., O’Brien, R., \& Krakauer, J. W. (2016). Paradoxical motor recovery from a first stroke after induction of a second stroke: reopening a postischemic sensitive period. Neurorehabilitation and Neural Repair, 30, 794-800. doi:10.1310/tsr1605-309 
Zorowitz, R. D., Chen, E., Bianchini Tong, K., \& Laouri, M. (2009). Costs and rehabilitation use of stroke survivors: a retrospective study of Medicare beneficiaries. Topics in Stroke Rehabilitation, 16, 309-320. doi:10.1177/1545968315624783 


\section{Tables}

Table 1

Response Times (RT, in ms), Percentage of Errors (PE, in \%) and Simon Effects for Trials in Block 1 and Block 2.

\begin{tabular}{|c|c|c|c|c|c|c|}
\hline \multirow[b]{2}{*}{ Trial } & \multicolumn{2}{|c|}{ Combined arms } & \multicolumn{2}{|c|}{ Affected arm } & \multicolumn{2}{|c|}{ Non-affected arm } \\
\hline & $\begin{array}{c}\mathrm{RT} \text { in } \\
\mathrm{ms}\end{array}$ & $\mathrm{PE}$ in $\%$ & $\mathrm{RT}$ in $\mathrm{ms}$ & $\mathrm{PE}$ in $\%$ & $\mathrm{RT}$ in $\mathrm{ms}$ & $\mathrm{PE}$ in $\%$ \\
\hline
\end{tabular}

\section{Block 1}

\begin{tabular}{lrrrrrr} 
Non-corresponding & $684(39)$ & $5.49(2.12)$ & $694(55)$ & $3.88(1.96)$ & $673(30)$ & $7.10(2.44)$ \\
$\begin{array}{l}\text { Corresponding } \\
\begin{array}{l}\text { Difference non- } \\
\text { corresponding } \\
\text { to corresponding } \\
\text { (Simon Effect) }\end{array}\end{array}$ & $655(43)$ & $1.87(0.79)$ & $670(61)$ & $1.66(0.07)$ & $641(30)$ & $2.09(0.97)$ \\
\hline
\end{tabular}

\section{Block 2: Critical Trials}

\begin{tabular}{lcccccc} 
Non-corresponding & $635(32)$ & $4.12(2.07)$ & $644(46)$ & $3.53(2.09)$ & $625(24)$ & $4.71(2.44)$ \\
$\begin{array}{l}\text { Corresponding } \\
\begin{array}{l}\text { Difference non- } \\
\text { corresponding } \\
\text { to corresponding } \\
\text { (Simon Effect) }\end{array}\end{array}$ & $629(36)$ & $1.67(0.81)$ & $641(53)$ & $3.35(1.61)$ & $617(23)$ & $0.00(0.00)$ \\
\hline
\end{tabular}

\section{Block 2: Control Trials}

\begin{tabular}{|c|c|c|c|c|c|c|}
\hline Non-corresponding & $662(41)$ & $8.25(2.94)$ & $674(56)$ & $10.62(2.99)$ & $649(28)$ & $5.88(2.20)$ \\
\hline Corresponding & $624(38)$ & $4.54(2.21)$ & $634(54)$ & $3.53(1.91)$ & $614(24)$ & $5.56(2.72)$ \\
\hline $\begin{array}{l}\text { Difference non- } \\
\text { corresponding } \\
\text { to corresponding } \\
\text { (Simon Effect) }\end{array}$ & $38(8)$ & $3.71(2.98)$ & $40(11)$ & $7.09(4.06)$ & $36(10)$ & $0.33(2.59)$ \\
\hline
\end{tabular}

Block 2: Difference Control vs. Critical Trials 
If-Then Plans Counteract Learned Non-Use

Difference non-

corresponding

to corresponding

32 (13) $1.27(2.29)$

$37(17)$

$6.91(3.59)$

$28(15) \quad 4.38(2.59)$

(Simon Effect)

Note. Standard errors are shown in parentheses. 
If-Then Plans Counteract Learned Non-Use

\section{Figure Captions}

Figure 1: Exemplary stimuli set for corresponding and non-corresponding trials based on goal instructions for left or right key-press responses. 


\section{Figures}

Figure 1

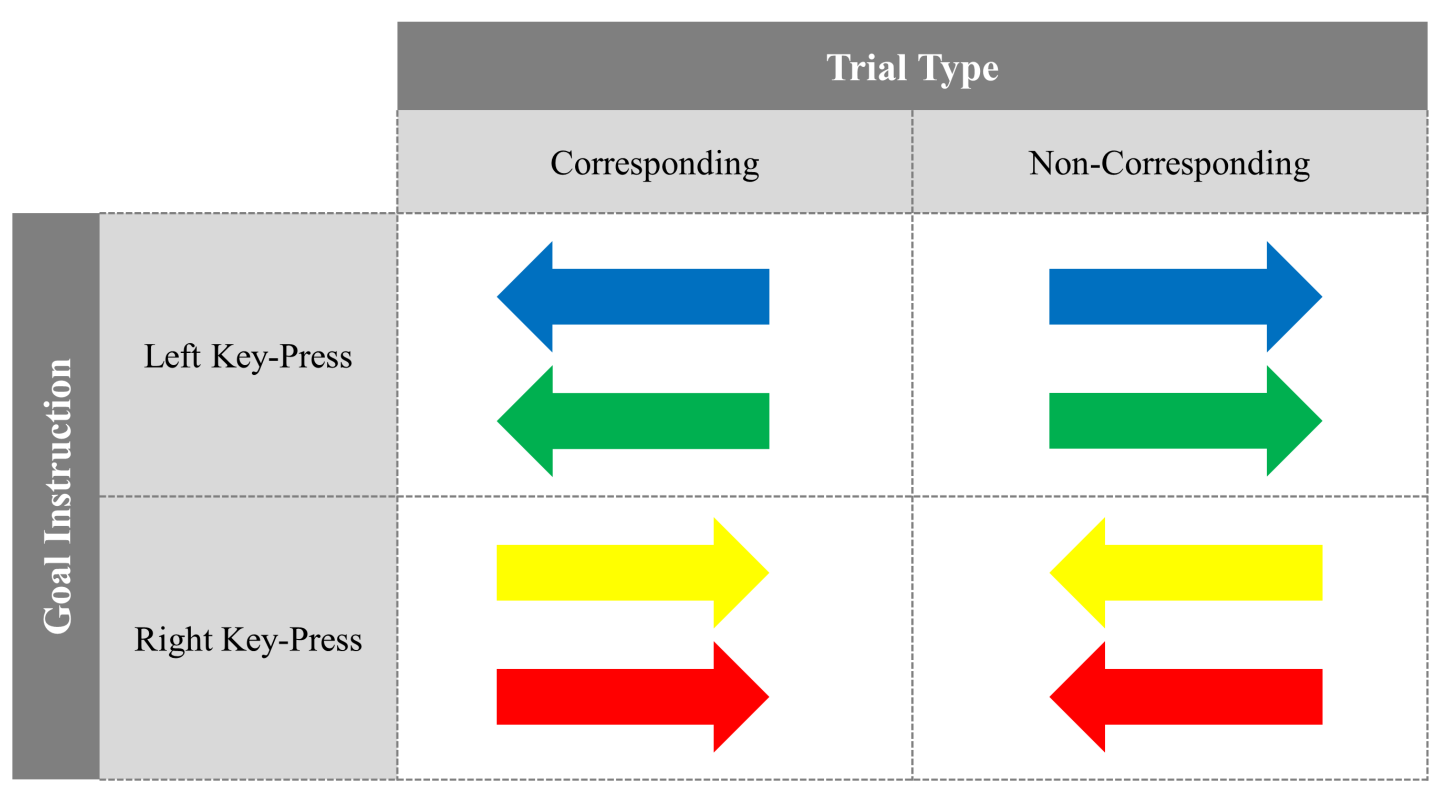

